\title{
Epigenetic programming of glucose-regulated insulin release
}

\author{
Frans Schuit \\ Department of Cellular and Molecular Medicine, Faculty of Medicine, KU Leuven, Leuven, Belgium.
}

\begin{abstract}
Pancreatic $\beta$ cells support glucose homeostasis with great precision by matching insulin release to the metabolic needs of the moment. Previous gene-expression analysis indicates that adult $\beta$ cells not only produce cellspecific proteins, but also repress a small set of housekeeping genes - such as those encoding lactate dehydrogenase A (LDHA), solute transporter MCT1, and hexokinase 1 (HK1) - that would otherwise interfere with normal $\beta$ cell function. In this issue of the JCI, Dhawan et al. elucidate a molecular mechanism involved in $\beta$ cell-specific repression of $L d h a$ and $H k 1$ that is mediated by induction of the de novo DNA methyltransferase DNMT3A during the first weeks after birth. Failure to induce DNMT3A-dependent methylation disrupts normal glucose-induced insulin release in adult life. The results of this study reinforce the idea that the phenotype of adult $\beta$ cells has two faces and that failure to achieve selective gene repression undermines $\beta$ cell support of normal glucose homeostasis.
\end{abstract}

unresponsive to a rise in extracellular pyruvate, a situation that is reversed in individuals with a Mendelian disorder called exercise-induced hyperinsulinism (9), which results from loss of regulation at the level of the human MCT1 promoter (10). The presence of MCT1 in $\beta$ cells would confuse the physiology of glucose homeostasis in that $\beta$ cells would no longer discriminate elevated glucose (after meals) from elevated pyruvate (after exercise) and would therefore fail to match circulating insulin levels to metabolic demands (Figure 1).

Although the number of genes specifically repressed in islets is low, the scale of the phenomenon increases when genes that are repressed not only in islets but also in one or two additional tissues are considered. One example is the group of low- $\mathrm{K}_{\mathrm{m}}$ hexokinases (HK1-HK3), which catalyze the first step of glycolysis in most cells. For pancreatic $\beta$ cells and hepatocytes, however, these isoforms are ill adapted to sustain glucose homeostasis because they are saturated by micromolar amounts of their substrate glucose. In contrast, glucokinase (GK, Figure 1) is well adapted for precise control of glucose homeostasis, as its enzymatic affinity for glucose is in the millimolar range (11). $\beta$ Cells efficiently repress HK1, and what little enzymatic activity that has been measured in islets was attributed to contaminating exocrine pancreatic cells (12). Forced expression of low- $\mathrm{K}_{\mathrm{m}}$ HK1 in $\beta$ cells (13) elevates basal insulin secretion, rendering $\beta$ cells dangerous in terms of hypoglycemic risk (Figure 1). Hk1, Ldha, and Mct1 therefore represent examples of a class of disallowed genes (14) that would jeopardize normal $\beta$ function when expressed (15).

\section{The start of an epigenetic roadmap}

How do $\beta$ cells selectively repress genes that are active in most other cell types? Epigenetic-based mechanisms have been suggested in earlier work. Thorrez et al. detected repressive chromatin markers in 
A

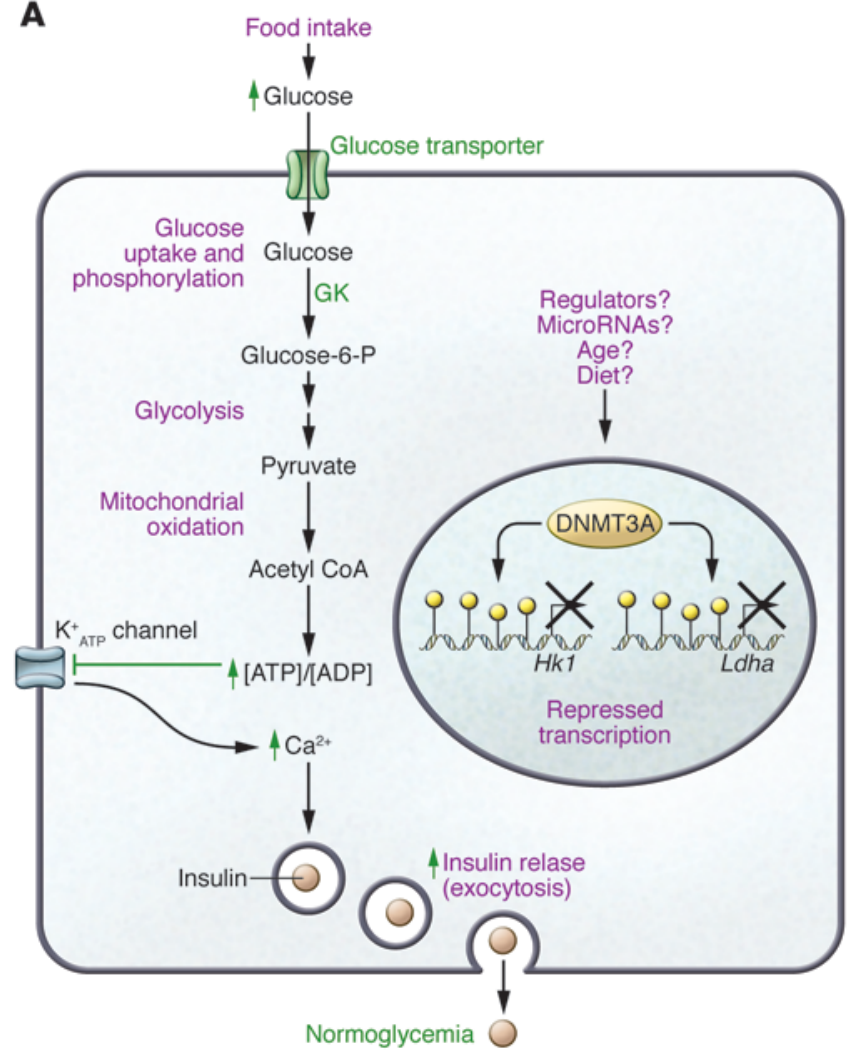

B

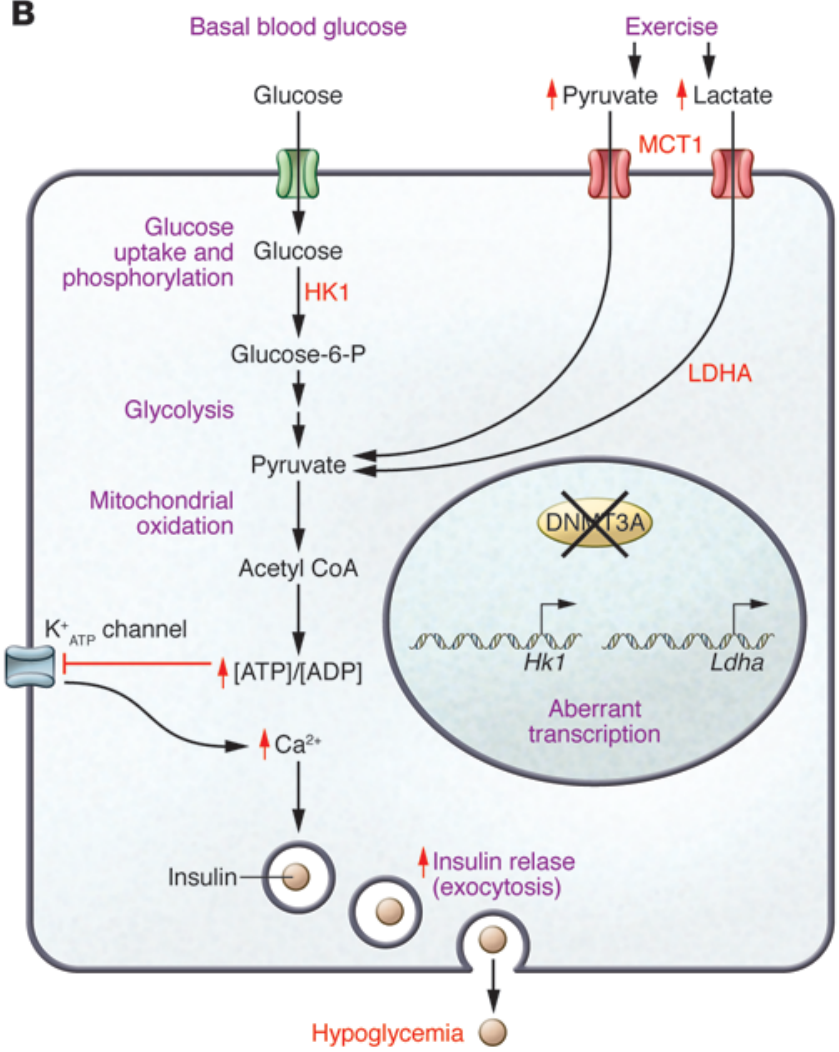

Figure 1. DNMT3A mediates repression of genes that disrupt normal glucose-regulated insulin release in $\boldsymbol{\beta}$ cells. (A) Physiological, nutrient-induced insulin release during a carbohydrate-containing meal depends on the expression of key proteins in pancreatic $\beta$ cells that couple a rise in circulating glucose to an accelerated metabolic flux, leading to an increased [ATP]/[ADP] ratio, closure of ATP-sensitive potassium channels and a rise in cytosolic calcium (1), and appropriate levels of insulin secretion that support normoglycemia. In this issue, Dhawan et al. (18) show that the DNA methyltransferase DNMT3A is critical for repression of disallowed genes $H k 1$ and $L d h a$ that would otherwise interfere with $\beta$ cell function. The factors that induce and maintain DNMT3A in $\beta$ cells have not been fully elucidated. (B) Inappropriate insulin secretion, as in too much insulin under basal conditions or activation of induction of secretion by nonphysiological stimuli, can cause hypoglycemia (15). In the absence of DNMT3A, expression of HK1 elevates basal insulin release because the enzyme has a high affinity for its substrate glucose, and elevated LDHA promotes conversion of lactate into pyruvate. Moreover, increased levels of the pyruvate transporter MCT1 promote inappropriate insulin secretion (10). Dhawan and colleagues show that murine $\beta$ cells switch off $H k 1$ and $L d h a$ expression when cells mature the first weeks after birth, as the result of DNMT3A-mediated cytosine methylation (yellow circles) in the Hk1 and Ldha gene promoters.

a few of the genes repressed in $\beta$ cells (4). A more systematic, genome-wide analysis of histone $\mathrm{H} 3$ modifications (16) showed that $\beta$ cell maturation is associated with both activation and inactivation of specific genes. In a follow-up study, the polycomb repressor complex subunit RING1B was identified as one of the molecular components that mediate these epigenetic changes (17).

In this issue (18), Dhawan and colleagues propose that the de novo DNA methyltransferase DNMT3A plays an important role in mediating epigenetic gene repression during $\beta$ cell maturation. Analysis of mRNA expression in FACS-purified $\beta$ cells from $M I P-G F P$ mice revealed that between postnatal days 4 and 25 (P4 and $\mathrm{P} 25)$, there is an upregulation of genes encoding key $\beta$ cell factors, including MAFA and GK (Figure 1), with a concurrent downregulation of $H k 1, H k 2, A l d o b$, and Ldha. Evaluation of methylated versus nonmethylated cytosines in the $H k 1$ and Ldha gene loci demonstrated that there is a net increase in methylation of these 2 loci between P4 and P25. ChIP analysis showed that the DNA methyltransferase DNMT3A is maximally enriched at the Ldha and Hk1 loci in $\beta$ cells two weeks after birth. Dhawan and colleagues developed a mouse strain with a $\beta$ cellspecific deletion of Dnmt3a (Dnmt3a $a^{\text {t/fl }}$ R26R-eYFP RIP-Cre mice, referred to as $3 a R C Y-\mathrm{KO})$ that allowed for direct evaluation for the role of DNMT3A in the methylation and repression of $H k 1$ and Ldha. Compared with controls, $3 a R C Y$-KO mice had elevated basal plasma insulin and a poor insulin response after i.p. glucose injection, and isolated islets from these animals were deficient for glucose-induced insulin release. The $H k 1$ and Ldha loci were weakly methylated in $\beta$ cells of $3 a R C Y-\mathrm{KO}$ mice, resulting in upregulation of Hk1 and Ldha transcripts. Moreover, siRNA-mediated knockdown of $H k 1$ and Ldha transcripts partially restored insulin secretory competence in $3 a R C Y$ -KO $\beta$ cells. Comparison of adult human pancreatic islets with immature, insulinexpressing cells derived from pluripotent cells revealed higher levels of DNA methylation and stronger repression of $H K 1$ and $L D H A$ in the mature islets. Together, the study by Dhawan et al. (18) supports the importance of disallowed gene repres- 
sion for normal $\beta$ cell function. Additionally, these results provide an explanation for how maturing $\beta$ cells switch off a set of genes that would destroy normal function when expressed and show that the de novo methyltransferase DNMT3A plays an active role in this process.

\section{New questions}

The study by Dhawan and colleagues on DNMT3A (18) and previous work on RING1B (17) can be viewed as starting points that provide important fragments of information for compiling a more complete epigenetic roadmap (19) of neonatal immature $\beta$ cells and adult mature $\beta$ cells. It will be interesting to elucidate how DNMT3A activity in $\beta$ cells is regulated and how the methyltransferase is guided toward its epigenetic target regions. One possibility is that histone H3K27 tri-methylation (H3K27me3), which is associated with polycomb repression, is a context-dependent chromatin signal that guides DNMT3A activity toward its relevant target genes (20). This raises the question of how H3K27me3 marks are selectively introduced in the disallowed target genes of maturing $\beta$ cells. Interestingly, not only were $H k 1$ and $L d h a$ upregulated in $3 a R C Y$ -KO $\beta$ cells, but expression of key $\beta$ cell genes, including $M a f a, P d x 1$, and $G k$, was reduced, indicating that these two phenomena are interconnected. It seems possible that some of the $\beta$ cell-specific transcription factors that enhance expression of a set of $\beta$ cell genes could also operate as repressors in a context-dependent manner. Future experiments should address the precise link between repression of disallowed genes and upregulation of $\beta$ cell genes.

It should also be noted that the neonatal period of DNMT3A activity is a continuation of intrauterine events that initiate $\beta$ cell identity via DNA methylation (21). This raises the question as to whether or not environmental conditions during pregnancy influence the starting position of neonatal epigenetic events. Moreover, what happens after $\beta$ cells have matured? How is the continued repression of disallowed genes at the end of the neonatal period maintained? Adult $\beta$ cells are essentially a nonreplicating population, yet they remain active during the entire lifetime of rodents and humans. Therefore, it seems relevant to assess the impact of environmental factors, such as a high-fat diet, that elevate basal insulin release. Because DNMT3A acts as a de novo methylase that introduces an epigenetic landscape in newly replicated cells (22), adult, nonreplicating, $\beta$ cells may utilize other mechanisms to maintain appropriate gene-expression patterns. For example, a cytosine demethylation pathway that is mediated by a family of Tet dioxygenases (23) can reactivate expression of methylated loci, but the activity of these enzymes has not been studied in adult $\beta$ cells. Future studies should also address a potential role for $\beta$ cell microRNA that may interfere with DNMT3A activity, in particular members of the miR-29 family (24).

It should be noted that Dhawan et al. (18) used the MIP-GFP and RIP-Cre mouse models, which harbor an artificial human growth hormone (hGH) minigene that is expressed in transgenic islets (25). Expression of the hGH minigene likely does not alter repression of disallowed genes, as hGH does not influence these genes in Pdx1-Cre mice (25). It is not clear why methylation at the $M c t 1$ promoter was not affected by Dnmt3a KO, as Mct1 has clear H3K27me3 marks in differentiated $\beta$ cells (4). Is it possible that complementary methyltransferases act on a different set of disallowed target genes?

Together, the study by Dhawan et al. (18) supports the earlier idea that $\beta$ cell differentiation has two faces (4): a visible face that shows accumulation of specific $\beta$ cell proteins and a hidden face that protects $\beta$ cells against ubiquitous proteins that need to be repressed to prevent disturbance of normal $\beta$ cell function. The facets of both faces need to be addressed in efforts to generate insulin-producing cells from pluripotent stem cells. Dhawan and colleagues (18) propose that DNMT3A is one of the waypoints in the epigenetic roadmap of $\beta$ cell maturation that is essential for the transition from immature to mature $\beta$ cells. Evaluation of the roles of DNMT3A in preventing $\beta$ cell abnormalities during chronic metabolic disease would appear to be interesting topics for future studies.

\section{Acknowledgments}

F. Schuit is currently supported by the Flemish Foundation for Scientific Research (FWO grant G067212N) for investigation of the phenomenon of disallowed genes in $\beta$ cells.
Address correspondence to: Frans Schuit, Department of Cellular and Molecular Medicine, Faculty of Medicine - KU Leuven, Herestraat 49 B3000 Leuven, Belgium. Phone: 32.16.330.849; E-mail: Frans.Schuit@med.kuleuven.be.

1. Rorsman P, Braun M. Regulation of insulin secretion in human pancreatic islets. Annu Rev Physiol. 2013;75:155-179.

2. Rutter GA, Pullen TJ, Hodson DJ, Martinez-Sanchez A. Pancreatic $\beta$-cell identity, glucose sensing and the control of insulin secretion. Biochem J. 2015;466(2):203-218.

3. Nishimura W, et al. A switch from MafB to MafA expression accompanies differentiation to pancreatic $\beta$-cells. Dev Biol. 2006;293(2):526-539.

4. Thorrez L, et al. Tissue-specific disallowance of housekeeping genes: the other face of cell differentiation. Genome Res. 2011;21(1):95-105.

5. Pullen TJ, Khan AM, Barton G, Butcher SA, Sun $G$, Rutter GA. Identification of genes selectively disallowed in the pancreatic islet. Islets. 2010;2(2):89-95.

6. Pullen TJ, Rutter GA. When less is more: the forbidden fruits of gene repression in the adult $\beta$-cell. Diabetes Obes Metab. 2013;15(6):503-512.

7. Sekine $\mathrm{N}$, et al. Low lactate dehydrogenase and high mitochondrial glycerol phosphate dehydrogenase in pancreatic $\beta$-cells. Potential role in nutrient sensing. J Biol Chem. 1994;269(7):4895-4902.

8. Schuit F, et al. Metabolic fate of glucose in purified islet cells. Glucose-regulated anaplerosis in $\beta$ cells. J Biol Chem . 1997;272(30):18572-18579.

9. Otonkoski T, et al. Physical exercise-induced hyperinsulinemic hypoglycemia is an autosomal-dominant trait characterized by abnormal pyruvate-induced insulin release. Diabetes. 2003;52(1):199-204.

10. Otonkoski T, et al. Physical exercise-induced hypoglycemia caused by failed silencing of monocarboxylate transporter 1 in pancreatic $\beta$ cells. Am J Hum Genet. 2007;81(3):467-474.

11. Matschinsky FM. Regulation of pancreatic $\beta$-cell glucokinase: from basics to therapeutics. Diabetes. 2002;51(suppl 3):S394-S404.

12. Schuit F, Moens K, Heimberg H, Pipeleers D. Cellular origin of hexokinase in pancreatic islets. J Biol Chem. 1999;274(46):32803-32809.

13. Becker TC, BeltrandelRio H, Noel RJ, Johnson $\mathrm{JH}, \mathrm{Newgard} \mathrm{CB}$. Overexpression of hexokinase I in isolated islets of Langerhans via recombinant adenovirus. Enhancement of glucose metabolism and insulin secretion at basal but not stimulatory glucose levels. J Biol Chem. 1994;269(33):21234-21238.

14. Quintens R, Hendrickx N, Lemaire K, Schuit F. Why expression of some genes is disallowed in $\beta$-cells. Biochem Soc Trans. 2008; 36(pt 3):300-305.

15. Schuit F, et al. $\beta$-Cell-specific gene repression: a mechanism to protect against inappropriate or maladjusted insulin secretion? Diabetes. 2012;61(5):969-975.

16. van Arensbergen J, et al. Derepression of Polycomb targets during pancreatic organogenesis 
allows insulin-producing $\beta$-cells to adopt a neural gene activity program. Genome Res. 2010;20(6):722-732.

17. van Arensbergen J, et al. Ring1b bookmarks genes in pancreatic embryonic progenitors for repression in adult $\beta$ cells. Genes Dev. 2013;27(1):52-63.

18. Dhawan S, et al. DNA methylation directs functional maturation of pancreatic $\beta$ cells. J Clin Invest. 2015;215(7):2851-2860.

19. Roadmap Epigenomics Consortium, et al. Integrative analysis of 111 reference human epigenomes.
Nature. 2015;518(7539):317-330.

20. Rose NR, Klose RJ. Understanding the relationship between DNA methylation and histone lysine methylation. Biochim Biophys Acta. 2014;1839(12):1362-1372.

21. Dhawan S, Georgia S, Tschen SI, Fan G Bhushan A. Pancreatic $\beta$ cell identity is maintained by DNA methylation-mediated repression of Arx Dev Cell. 2011;20(4):419-429.

22. Okano M, Bell DW, Haber DA, Li E. DNA methyltransferases Dnmt3a and Dnmt3b are essential for de novo methylation and mammalian development. Cell.1999;99(3):247-257.

23. He YF, et al. Tet-mediated formation of 5-carboxylcytosine and its excision by TDG in mammalian DNA. Science. 2011;333(6047):1303-1307.

24. Fabbri M, et al. MicroRNA-29 family reverts aberrant methylation in lung cancer by targeting DNA methyltransferases 3A and 3B. Proc Natl Acad Sci U S A. 2007;104(40):15805-15810.

25. Brouwers B, et al. Impaired islet function in commonly used transgenic mouse lines due to human growth hormone minigene expression. Cell Metab. 2014;20(6):979-990. 